\title{
PRÁTICA BASEADA EM EVIDÊNCIA NO CONTEXTO DOS NÚCLEOS DE APOIO A SAÚDE DA FAMÍLIA EM CHAPECÓ
}

\author{
Fátima Ferretti ${ }^{1}$, Fabiana Romancini², Luana Roberta Schneider ${ }^{3}$, Lucimare Ferraz ${ }^{4}$
}

\begin{abstract}
RESUMO: Objetivo: analisar a Prática Baseada em Evidência no trabalho dos profissionais da equipe do Núcleo de Apoio à Saúde da Família. Método: estudo descritivo realizado no município de Chapecó - Santa Catarina. A população do estudo foi composta de 28 profissionais que atuavam nos núcleos, no ano de 2016. A análise dos dados ocorreu por estatística descritiva. Resultados: os profissionais destacaram dificuldades em converter as necessidades de conhecimento em questão de pesquisa, mas se disseram aptos para levantar evidências científicas e identificar os principais tipos e fontes de informação existentes. Entre os obstáculos para o desenvolvimento da Prática Baseada em Evidência, mencionaram a alta carga de trabalho e a falta de incentivo dos gestores. Conclusão: os profissionais necessitam se apropriar dos elementos da Prática Baseada em Evidência, auxiliando as equipes na resolução dos problemas de saúde.
\end{abstract}

DESCRITORES: Atenção primária à saúde; Prática clínica baseada em evidências; Pessoal de saúde: Saúde da família; Serviços de saúde.

\section{EVIDENCE-BASED PRACTICE IN THE CONTEXT OF THE FAMILY HEALTH SUPPORT CENTERS IN CHAPECÓ}

ABSTRACT: Objective: to analyze Evidence-Based Practice in the work of the professionals of the Family Health Support Center team. Method: descriptive study carried out in the municipality of Chapecó - Santa Catarina. The study population consisted of 28 professionals working in the centers in the year 2016. Data analysis was performed through descriptive statistics. Results: the professionals highlighted difficulties in converting knowledge needs into research questions, however, reported that they were able to collect scientific evidence and identify the main types and sources of information existing. Among the obstacles to the development of Evidence-Based Practice, they mentioned the high workload and lack of incentives from the managers. Conclusion: the professionals need to appropriate the elements of Evidence-Based Practice, helping the teams in the resolution of health problems.

KEYWORDS: Primary healthcare; Evidence-based clinical practice; Health personnel: Family health; Health services.

\section{PRÁCTICA BASADA EN EVIDENCIA EN NÚCLEOS DE APOYO A SALUD DE LA FAMILIA EN CHAPECÓ}

RESUMEN: Objetivo: analizar la Práctica Basada en Evidencia en el trabajo de profesionales del equipo del Núcleo de Apoyo a Salud de la Familia. Método: estudio descriptivo realizado en municipio de Chapecó - Santa Catarina. Población integrada por 28 profesionales actuantes en los núcleos, durante 2016. Datos analizados por estadística descriptiva. Resultados: los profesionales destacaron dificultades para convertir las necesidades de conocimiento que fueron objeto de la investigación, aunque afirmaron estar aptos para relevar evidencias científicas e identificar los principales tipos de fuentes de información existentes. Entre los obstáculos para el desarrollo de la Práctica Basada en la Evidencia, mencionaron la alta carga de trabajo y falta de estímulo de los gestores. Conclusión: los profesionales necesitan apropiarse de los elementos de la Práctica Basada en Evidencias, ayudando a los equipos en la resolución de los problemas de salud.

DESCRIPTORES: Atención Primaria de Salud; Práctica Clínica Basada en la Evidencia; Personal de Salud; Salud de la Familia; Servicios de Salud.

\footnotetext{
${ }^{1}$ Fisioterapeuta. Doutora em Saúde Coletiva. Docente do Programa de Pós-graduação Stricto Sensu em Ciências da Saúde da Universidade Comunitária da Região de Chapecó-Unochapecó. Chapecó-SC-Brasil.

${ }^{2}$ Acadêmica do curso de medicina da Universidade Comunitária da Região de Chapecó-Unochapecó. Chapecó-SC-Brasil.

${ }^{3}$ Enfermeira. Mestre em Ciências da Saúde. Docente do Curso de Enfermagem e Medicina da Universidade Comunitária da Região de Chapecó-Unochapecó. Chapecó-SC-Brasil.

${ }^{4}$ Enfermeira. Doutora em Saúde Coletiva. Docente do Programa de Pós-graduação Stricto Sensu em Ciências da Saúde da Universidade Comunitária da Região de Chapecó-Unochapecó. Chapecó-SC-Brasil.
} 


\section{INTRODUÇÃO}

A partir da criação do Sistema Único de Saúde (SUS), desenhou-se uma nova forma de organização dos serviços e práticas em saúde, fundamentada na Atenção Básica ${ }^{(1)}$. A Atenção Básica (AB) configurase como a porta de entrada para o sistema de saúde e representa o conjunto de ações de promoção e proteção da saúde, prevenção de agravos, diagnóstico, tratamento, reabilitação e manutenção da saúde em âmbito individual e coletivo(2).

Com a finalidade de melhorar a qualidade e a abrangência da Atenção Básica, criou-se o Núcleo de Apoio à Saúde da Família (NASF), pela Portaria n. ${ }^{\circ} 154$ de 24 de janeiro de $2008^{(3)}$. O trabalho nos NASF contribui diretamente para a transferência de saberes, capacitações e responsabilidades, originando experiência para todos profissionais dos núcleos. Basicamente, o NASF é uma estratégia para melhorar a eficiência e a resolutividade das equipes quando estas atuam frente aos problemas de saúde da população(4).

As atividades comuns a todos os profissionais envolvidos no núcleo são desenvolvidas em conjunto com as equipes de saúde da família e são pautadas por acolhimento, humanização da saúde, intersetorialidade, ações multiprofissionais e responsabilidade compartilhada ${ }^{(5)}$. A complexidade das ações desenvolvidas pelos profissionais que atuam nesse cenário exige-lhes capacidade de análise, intervenção e autonomia para a fixação de práticas transformadoras, gestão das mudanças e o estreitamento dos elos entre a compreensão e a execução do trabalho(2).

Entre as diversas características e finalidades do NASF, destacam-se a equipe formada por diferentes profissionais, o apoio especializado na própria Atenção Básica, o suporte em situações previstas e imprevistas, as ações compartilhadas com as equipes da Estratégia Saúde da Família (ESF), o auxílio à equipe para evitar os encaminhamentos realizados a outros pontos de atenção, o aumento da capacidade da equipe na Atenção Básica e o auxílio na articulação com outros pontos de atenção na rede ${ }^{(4)}$.

O NASF deve atuar em articulação com a ESF com o intuito de fortalecer o trabalho em equipe, realizando o cuidado especializado a usuários, bem como deve exercer o feedback, a discussão e a educação permanente ${ }^{(6)}$. Para isso, é necessário que os profissionais utilizem a Prática Baseada em Evidência (PBE) como estratégia para ampliar a qualidade de suas práticas e a resolubilidade da ESF.

A Prática Baseada em Evidência tem se constituído em ferramenta para qualificar o trabalho em saúde, além de se apresentar como boa estratégia para a efetividade clínica e o apoio para a tomada de decisão, que o profissional assume ao desempenhar o cuidado $^{(7)}$. Essa prática baseia-se na delimitação de um problema e na tomada de decisão pautada no conhecimento, já publicado sobre o assunto, somado ao conhecimento obtido na prática ${ }^{(8)}$. Para outros autores, a PBE é a integração entre as melhores evidências científicas, a habilidade clínica e as preferências do paciente ${ }^{(9)}$. Ainda, tal prática configura-se como um campo de conhecimento permanente e em constante construção, para análise e maior compreensão de fenômenos em saúde(10).

Assim sendo, a PBE é importante ferramenta para qualificação da assistência à saúde ${ }^{(11)}$, bem como para transpor a dicotomia entre a pesquisa e o cuidar ${ }^{(12)}$. Não obstante, observa-se a ausência de estudos sobre o uso da PBE na prática desenvolvida pela equipe do NASF. A maioria das pesquisas feitas no Brasil, considerando a PBE, é sobre infância e adolescência, infectologia, saúde mental e cirurgia, tendo como enfoques predominantes a prevenção, o tratamento, a reabilitação, o diagnóstico e a avaliação(11). Considerando o exposto, o objetivo deste estudo foi analisar a PBE no trabalho dos profissionais da equipe do NASF no município de Chapecó - Santa Catarina (SC). 


\section{METODOLOGIA}

Trata-se de uma pesquisa quantitativa, de caráter descritivo e transversal. O cenário da pesquisa contemplou todas as unidades de saúde do município de Chapecó - SC que possuíam equipe do NASF atuando no ano de 2016. De acordo com os dados de acompanhamento da Atenção Básica da Secretaria de Estado de Saúde, o município possui cinco NASF da modalidade I, sendo denominados de NASF Oeste, NASF Sul, NASF Leste, NASF Norte e NASF Centro ${ }^{(13)}$.

Todos os profissionais pertencentes ao NASF foram convidados para participar da pesquisa. De acordo com informações obtidas da Secretaria Municipal de Saúde em março de 2016, os profissionais cadastrados no NASF eram: dois fisioterapeutas, cinco psicólogos(as), cinco farmacêuticos(as), cinco nutricionistas, cinco educadores(as) físicos(as) e seis assistentes sociais, totalizando 28 profissionais $^{(13)}$. Entre todos os profissionais do NASF, foram incluídos aqueles que atuavam no núcleo há mais de 6 meses e que aceitaram participar do estudo. Foram excluídos dois participantes que no momento da coleta estavam de licença-maternidade, um que após três tentativas de contato não se disponibilizou a responder ao questionário, e três profissionais que não atuavam mais no NASF (demissão durante o período), totalizando uma amostra de 22 profissionais.

A coleta de dados ocorreu entre os meses de julho e agosto de 2016 e deu-se por meio de dois instrumentos com questões objetivas: um Questionário de Prática Baseada em Evidências e Efetividade Clínica (EBPQ), instrumento validado para a versão brasileiraa ${ }^{(14)}$, composto de três partes relacionadas com atitudes, conhecimentos e habilidades associados à PBE; e um Roteiro de Questões Auxiliares (RQA), desenvolvido pelas pesquisadoras mediante estudos anteriores.

O RQA contém 14 questões objetivas. No primeiro bloco, as questões estão relacionadas às características dos participantes, como sexo, idade, ano de formação da graduação, ter ou não pósgraduação e tempo de atuação na Atenção Primária à Saúde (APS). O segundo bloco diz respeito à realização de pesquisas e ao acesso a recursos no ambiente de trabalho, como computador e Internet. No terceiro bloco, as questões buscam identificar os tipos de fontes de informação e a frequência da PBE na prática assistencial. Por fim, o último bloco relaciona-se com a gestão, o domínio de língua estrangeira e a relação ensino-serviço.

A análise dos dados foi realizada digitando-se primeiramente as variáveis numa planilha do programa Excel, que posteriormente foi transferida para o software Statistical Package for the Social Sciences (SPSS®), versão 22.0. Neste programa, foram efetuados cálculos de medidas de posição (média, mínima e máxima) e de dispersão (desvio-padrão). A pesquisa foi aprovada pelo Comitê de Ética em Pesquisa da Universidade Comunitária da Região de Chapecó, sob o parecer n.ํㅜ 1.573.371.

\section{RESUltados}

Entre os profissionais do NASF que participaram da pesquisa, havia um fisioterapeuta, quatro psicólogos(as), quatro farmacêuticos(as), quatro nutricionistas, cinco educadores(as) físicos(as) e quatro assistentes sociais.

Do total de participantes, verificou-se que $81,8 \%(n=18)$ eram mulheres, com média de idade de 31,3 anos (DP $\pm 7,44$ anos), idade mínima de 23 e máxima de 56 anos. A média de tempo de formação foi de 8 anos (DP $\pm 6,81)$, mediana de seis (3-33) e 90,9\% ( $n=20)$ possuíam pós-graduação, sendo todos pós-graduados em especialização. A média de tempo que os profissionais do estudo trabalhavam na Atenção Básica foi de 3 anos $\left(\mathrm{DP}_{ \pm 2}\right.$,15) e mediana de quatro, com mínima de 1 e máximo de 8 anos.

De acordo com os profissionais do NASF, em suas práticas na APS, a experiência clínica é o principal norteador de suas ações, com média de 5,59. Já a preferência do paciente nas tomadas de decisões evidenciase como o componente mais tênue no contexto dos pilares da PBE, com média de 4,14 (Tabela 1). 
Tabela 1 - Frequência que os profissionais do NASF consideram os elementos da Prática Baseada em Evidência na Atenção Primária à Saúde. Chapecó, SC, Brasil, 2017

\begin{tabular}{lcc}
\hline \multicolumn{1}{c}{ Variáveis* $^{*}$} & Média \pm DP & Mediana (mín.; máx.) \\
\hline A sua experiência clínica & $5,59 \pm 1,14$ & $6(3 ; 7)$ \\
\hline As evidências científicas & $5,23 \pm 1,23$ & $5(3 ; 7)$ \\
\hline A preferência do paciente & $4,14 \pm 1,28$ & $4(1 ; 6)$ \\
& & \\
\hline
\end{tabular}

*Respostas apresentadas numa escala Likert de 1 (nunca) até 7 (frequentemente).

DP= desvio-padrão; Mín.= mínima; Máx.= máxima.

A Tabela 2 caracteriza os conhecimentos e habilidades dos profissionais do NASF para o desenvolvimento da PBE, numa escala Likert que varia de 1 a 7. Quanto às habilidades para rever a prática e compartilhar ideias, observou-se uma média de 4,91. Quanto à habilidade em pesquisa, evidencia-se maior dificuldade em converter as necessidades de conhecimento em questão de pesquisa, verificandose uma média de 4,41. Já em relação aos conhecimentos, os profissionais apresentaram média favorável para levantar evidências $(4,64)$ e para identificar os principais tipos e fontes de informação existentes $(5,18)$.

Tabela 2 - Apresentação da escala sobre os conhecimentos e habilidades dos profissionais do NASF para a PBE. Chapecó, SC, Brasil, 2017

\begin{tabular}{llc}
\hline Variáveis* & Média \pm DP & $\begin{array}{c}\text { Mediana } \\
\text { (mín.; máx.) }\end{array}$ \\
\hline Conhecimentos para: & & \\
\hline Identificar os principais tipos e fontes de informação existentes & $5,18 \pm 0,85$ & $5(4 ; 7)$ \\
\hline Levantar evidências & $4,64 \pm 1,09$ & $5(3 ; 6)$ \\
\hline Habilidades para: & $5,36 \pm 0,95$ & $5,5(4 ; 7)$ \\
\hline Rever a sua própria prática & $5,23 \pm 1,27$ & $5,5(2 ; 7)$ \\
\hline Compartilhar suas ideias e conhecimento com os colegas de trabalho & $5,09 \pm 1,34$ & $5,5(2 ; 7)$ \\
\hline Disseminar novas ideias sobre cuidado entre os colegas & $5,00 \pm 1,02$ & $5(3 ; 7)$ \\
\hline Identificar lacunas na prática profissional & $5,41 \pm 1,14$ & $5,5(3 ; 7)$ \\
\hline Aplicar o conhecimento a casos individuais & $5,18 \pm 1,26$ & $5(3 ; 7)$ \\
\hline Utilizar-se da informática & $5,18 \pm 1,01$ & $5(3 ; 7)$ \\
\hline Determinar quão aplicável clinicamente é o material & $4,73 \pm 1,12$ & $5(3 ; 7)$ \\
\hline Monitorar e revisar as práticas & $4,91 \pm 1,07$ & $5(3 ; 7)$ \\
\hline Realizar pesquisa & $5,32 \pm 0,89$ & $5(3 ; 7)$ \\
\hline Determinar quão válido é o material & $4,91 \pm 1,07$ & $5(2 ; 6)$ \\
\hline Analisar criticamente as evidências frente aos padrões já estabelecidos & $4,41 \pm 1,44$ & $4(2 ; 7)$ \\
\hline Converter suas necessidades de conhecimento em uma questão de pesquisa & $4,1,19$ \\
\hline
\end{tabular}

*Respostas apresentadas numa escala Likert de 1 (ruim) até 7 (ótimo). DP= desvio-padrão; Mín.= mínima; Máx.= máxima.

Quando observados os resultados do RQA, verificou-se que durante a formação profissional 90,9\% $(n=20)$ foram capacitados a buscar artigos científicos. Contudo, somente $77,3 \%(n=17)$ solucionaram suas dúvidas por intermédio de revistas científicas, $54,5 \%(n=12)$ pela Internet e $50 \%(n=11)$ pelo Telessaúde. Além disso, pode-se reiterar que a quantidade de profissionais do NASF que usam a Internet 
para solucionar dúvidas é baixa, já que todos possuem computador com acesso à rede disponível na unidade de saúde.

Observa-se que apenas três $(13,6 \%)$ profissionais leem e compreendem "Bem" a língua Inglesa, e quatro $(18,2 \%)$ leem e dois $(9,1 \%)$ compreendem "Bem" a língua Espanhola.

Na Tabela 3, os dados evidenciam que os profissionais do NASF reconhecem que as evidências científicas são fundamentais para o exercício da sua profissão e que podem transformar sua prática. Todavia, demonstraram que a carga de trabalho na APS é um obstáculo para permanecerem atualizados com todas as novas evidências científicas (Tabela 3).

Tabela 3 - Descrição das atitudes dos profissionais do NASF frente à PBE. Chapecó, SC, Brasil, 2017

\begin{tabular}{|c|c|c|}
\hline Variáveis* & $\begin{array}{c}\text { Média } \pm \text { DP } \\
\text { Mediana (mín.; máx.) }\end{array}$ & Variáveis* \\
\hline $\begin{array}{l}\text { Minha carga de trabalho é muito } \\
\text { grande para que eu me mantenha } \\
\text { atualizado com todas as novas } \\
\text { evidências. }\end{array}$ & $\begin{array}{l}\square \square \mathbf{\square} \square \square \\
4,14 \pm 2,01 \\
4(1 ; 7)\end{array}$ & $\begin{array}{l}\text { Novas evidências são tão importantes que eu } \\
\text { defino um tempo para isso na minha agenda } \\
\text { de trabalho. }\end{array}$ \\
\hline $\begin{array}{l}\text { Eu me sinto desconfortável quando } \\
\text { minha prática é questionada. }\end{array}$ & $\begin{array}{c}\square \square \square \square \square \mathbf{\square} \\
5,64 \pm 1,53 \\
6(1 ; 7) \\
\end{array}$ & $\begin{array}{l}\text { Eu acolho de forma aberta os questionamentos } \\
\text { sobre a minha prática. }\end{array}$ \\
\hline $\begin{array}{l}\text { Práticas baseadas em evidências } \\
\text { são perda de tempo. }\end{array}$ & $\begin{array}{c}\square \square \square \square \square \square \mathbf{\square} \\
6,59 \pm 0,85 \\
7(4 ; 7) \\
\end{array}$ & $\begin{array}{l}\text { Práticas baseadas em evidências são } \\
\text { fundamentais para a prática profissional. }\end{array}$ \\
\hline $\begin{array}{l}\text { Eu mantenho o uso de métodos } \\
\text { testados e confiáveis ao invés de } \\
\text { mudar para algo novo. }\end{array}$ & $\begin{array}{c}\square, 59 \pm 1,10 \\
6(4 ; 7) \\
\end{array}$ & $\begin{array}{l}\text { Minha prática tem mudado em função das } \\
\text { evidências que tenho encontrado. }\end{array}$ \\
\hline
\end{tabular}

*Respostas apresentadas numa escala Likert de 1 (ruim) até 7 (ótimo). DP= desvio-padrão; Mín.= mínima; Máx.= máxima.

Os profissionais do NASF, quando questionados sobre a existência de incentivo dos gestores para momentos de estudos durante a jornada de trabalho, apresentaram uma média de 4,64 na escala Likert de 1 a 7 . Observou-se também que 45,5\% (n=10) desenvolveram alguma pesquisa, com ou sem a equipe, nos últimos 3 anos. Foi constatado que quatro $(18,2 \%)$ dos profissionais do NASF têm vínculo (tutoria, supervisão direta ou indireta, entre outros), em seu ambiente de trabalho, com pessoas de alguma universidade, sendo que três $(13,6 \%)$ são acadêmicos.

\section{DISCUSSÃO}

Embora os profissionais do NASF participantes desta pesquisa reconheçam a importância de realizar a PBE, esta ainda é muito centrada no pilar da experiência clínica e em menor escala nos pilares da evidência científica e da preferência do paciente. Um estudo realizado em São Paulo (SP) também evidenciou que significativa parcela dos profissionais da área da saúde considera a opinião dos especialistas a mais importante para a tomada de decisão ${ }^{(15)}$.

Além de considerar o conhecimento dos especialistas, é relevante que os profissionais do NASF ampliem o uso da PBE no NASF, pois quando a equipe compartilha opiniões e saberes na tentativa de promover o cuidado do indivíduo, como propõe o Projeto Terapêutico Singular (PTS), qualifica o projeto e as propostas das ações ${ }^{(5)}$. Ademais, entende-se que o NASF tem como função trazer evidências científicas para dialogar com a ESF, uma vez que atua na Atenção Básica como uma fonte de educação continuada. 
Ouvir o paciente e considerar as suas opiniões e crenças também faz parte do processo da PBE. Da mesma maneira, sem o uso da melhor evidência científica, o cuidado torna-se ultrapassado, e a probabilidade de erros na conduta aumenta, uma vez que a PBE tem como objetivo final o melhor resultado para o paciente, dentro dos recursos disponíveis ${ }^{(16)}$.

Assim como em estudos anteriores, os quais afirmam que conhecimento e habilidades insuficientes são barreiras significativas à $\mathrm{PBE}^{(17)}$, os resultados desta investigação apontam que os profissionais do NASF apresentam dificuldades em converter suas necessidades de conhecimento em uma questão de pesquisa e, consequentemente, ancoram-se em protocolos disponibilizados pelo Ministério da Saúde para tomada de decisão.

O fato de os profissionais terem como suporte os protocolos se deve também à disponibilização desses materiais, pelo Ministério da Saúde, para nortear as ações do NASF. Essa prática é mensurada pela Autoavaliação para Melhoria do Acesso e da Qualidade da Atenção Básica (AMAQ - NASF), que avalia se a coordenação disponibiliza mecanismos presenciais e/ou virtuais, como comunidades de práticas, Telessaúde, grupos de discussão, sítio virtual interativo ou rede social colaborativa, seminários, mostras, oficinas, entre outros, a fim de promover o compartilhamento de experiências e a troca de conhecimentos $^{(18)}$.

Utilizado para solucionar dúvidas e resolver problemas pela metade dos profissionais estudados nesta pesquisa, o Telessaúde é considerado uma estratégia para a qualificação da Atenção Primária à Saúde (APS). Suas ações são direcionadas a todos os profissionais que trabalham na APS e aos demais profissionais dos Núcleos de Apoio à APS. Seu objetivo é qualificar o trabalho das equipes que atuam no eixo da APS, e suas atividades são orientadas pelos princípios do SUS, pelos atributos da APS e pela melhor e mais atual evidência científica disponível adequada à prática da atenção primária ${ }^{(19)}$.

Embora os protocolos sejam instrumentos validados e seu uso incentivado pelo Ministério da Saúde, suas condutas são verticalizadas, ou seja, não consideram aspectos epidemiológicos e culturais das diversas regiões do Brasil. Também não há segurança se os conteúdos desses protocolos se baseiam nas melhores evidências científicas. Os protocolos ou guidelines são ferramentas da gestão altamente respeitadas, e a análise acerca dos seus limites é de que eles podem promover uma alienação no trabalho quando passam a ser usados indiscriminadamente como padrões, pois se perde a perspectiva originária de sua formulação ${ }^{(20)}$.

Os profissionais não necessitam excluir o uso dos protocolos de suas práticas, mas devem considerar o território e o contexto do paciente. Boa parte dos profissionais de saúde, nos dias de hoje, ainda apresenta um ideal positivista, impondo um modelo teórico fechado, em que o usuário do serviço não participa ativamente do processo, além de dissociar a saúde e a doença dos aspectos e dimensões históricas, sociais e culturais dos indivíduos ${ }^{(21)}$.

No entanto, isso não significa que os profissionais da saúde devam deixar de lado os modelos teóricos que os direcionam em suas atividades, mas precisam se comunicar com os outros para conhecer as reais necessidades do indivíduo. Além disso, devem levar em conta que são sujeitos de sua própria história, e que, portanto, exercem controle sobre suas vidas, percebendo e agindo segundo experiências na vida coletiva $^{(21)}$.

Outro resultado deste estudo diz respeito à formação acadêmica, demonstrando que, apesar de os profissionais demarcarem que foram capacitados em suas formações acadêmicas a buscar artigos científicos, a maioria não o faz. Essa atitude de não buscar produções científicas é um fator limitador, tanto pela privação de formação direcionada para a abordagem da PBE quanto pela ausência de continuidade de incentivo do processo de investigação após a graduação. Para formar estudantes com aspiração para a pesquisa é necessário desenvolver condições favoráveis durante todo o período da graduação. Nesse sentido, é primordial incentivar os acadêmicos e envolvê-los nas atividades de pesquisa e extensão das universidades $^{(22)}$. Em pesquisa semelhante, realizada com fisioterapeutas do estado de São Paulo, Brasil, que objetivou identificar conhecimentos e habilidades em relação à PBE, os profissionais reconheceram que a informação adquirida durante a graduação foi insuficiente para a implantação da PBE ${ }^{(15)}$. 
Neste estudo, os profissionais do NASF apresentam como fator limitador da PBE a carga de trabalho elevada. Outro autor afirma que a sobrecarga do NASF, com excesso de usuários e tarefas da ESF com grupos e ações generalistas, ocorre em função da baixa qualidade de algumas ESF em filtrar os usuários e/ou das poucas ou inexistentes ações coletivas cabíveis (generalistas)(6). Estudos semelhantes afirmam que a falta de tempo é a barreira pessoal mais comum para que os profissionais de saúde implantem a $\mathrm{PBE}^{(15,17)}$. Não obstante os profissionais da área da saúde apresentarem atitudes positivas frente à necessidade de basear seu trabalho e implantar a PBE, pesquisas apontam que estes não se sentem habilitados para fazer mudanças em sua prática ${ }^{(23-24)}$.

Ainda que o desenvolvimento de pesquisas e da PBE seja considerado uma atividade fundamental para a prática dos profissionais do NASF investigados neste estudo, a integração de evidências científicas com a atualização da prática clínica aparenta ser um processo desafiador nos serviços de saúde ${ }^{(25)}$. Assim sendo, pode-se proferir que as atitudes dos profissionais do NASF não dependem unicamente de suas posturas, uma vez que elas também são normatizadas pela organização e política dos serviços de saúde. Não obstante, a iniciativa para mudar essa realidade precisa ser exercida por seus interessados, os profissionais do NASF. Concorda-se que essa transformação não é algo simplista, já que os aspectos de formação, organização dos serviços e incentivos dos órgãos de representação profissional carecem de revisão e aprimoramento.

O NASF deve ser mais que um usuário de evidências científicas, deve ser também um gerador, de forma a complementar as ações da ESF. Outro autor justifica a articulação entre o NASF e a ESF ao apontar que ações clínicas e de trabalho devem ocorrer de forma sinérgica, desenvolvendo educação permanente mútua e oferecendo educação permanente à ESF, com aumento da resolubilidade, já que o NASF é estratégia para melhorar a eficiência e a resolutividade dos problemas de saúde na Atenção Básica $^{(6)}$. Os trabalhos dos NASF contribuem diretamente para a transferência de saberes, capacitações e responsabilidades, originando experiência para todos profissionais dos núcleos ${ }^{(4)}$.

Este estudo tem a limitação de ter sido realizado em um único município, mas considerado de referência regional em saúde para o estado de Santa Catarina. Apesar disso, esta investigação traz informações que podem subsidiar reflexões quanto à atuação do Núcleo de Apoio à Saúde da Família em outras regiões e municípios, pois identifica as lacunas de conhecimentos e habilidades dos profissionais que nele trabalham em relação ao ato de fazer e utilizar as pesquisas científicas para subsidiar a tomada de decisão. O presente estudo também estimula o debate em torno da necessidade de fortalecer o processo de formação profissional para uma Prática Baseada em Evidência.

\section{CONCLUSÃO}

Os profissionais do NASF reconheceram que a PBE é fundamental para o exercício da sua profissão, mas que atualmente está mais centrada nas experiências clínicas do profissional e em menor escala nos pilares da evidência científica.

A equipe da ESF, pela sua função generalista, necessita de apoio especializado para atender à demanda. Assim, é função do NASF esse cuidado especializado, e, por isso, é necessário que haja revisão dos processos de trabalho, uma vez que a carga de trabalho não pode ser entrave para o NASF utilizar a PBE para realizar sua função, que é oferecer apoio especializado à equipe de ESF.

Contudo, pesquisas são necessárias para compreender e reconhecer possíveis barreiras no intuito de consolidar a PBE, tendo em vista que o desenvolvimento dessa abordagem possibilita aos serviços de saúde assistir as pessoas de forma mais eficiente e resolutiva. 


\section{REFERÊNCIAS}

1. Ministério da Saúde (BR). Secretaria de Atenção à Saúde. Departamento de Atenção Básica. Política Nacional de Atenção Básica. [Internet] Brasília: Ministério da Saúde, 2012. [acesso em 12 dez 2017]. Disponível: http://189.28.128.100/dab/ docs/publicacoes/geral/pnab.pdf.

2. Ministério da Saúde. Portaria no 2.488, de 21 de outubro de 2011. Aprova a Política Nacional de Atenção Básica, estabelecendo a revisão de diretrizes e normas para a organização da Atenção Básica, para a Estratégia Saúde da Família (ESF) e o Programa de Agentes Comunitário. Diário Oficial da União, 21 out 2011.

3. Ministério da Saúde. Portaria no 154, de 24 de janeiro de 2008. Cria os Núcleos de Apoio à Saúde da Família - NASF. Diário Oficial da União, 25 jan 2008.

4. Ministério da Saúde (BR). Secretaria de Atenção à Saúde. Departamento de Atenção Básica. Núcleo de Apoio à Saúde da Família - Volume 1: ferramentas para a gestão e para o trabalho cotidiano. Cadernos de Atenção Básica n. 39. [Internet] Brasília: Ministério da Saúde; 2014 [acesso em 15 mai 2017]. Disponível: http://bvsms.saude.gov.br/bvs/ publicacoes/nucleo_apoio_saude_familia_cab39.pdf.

5. Ministério da Saúde (BR). Secretaria de Atenção à Saúde. Departamento de Atenção Básica. Cadernos de atenção básica. Diretrizes do NASF: núcleo de apoio à saúde da família. [Internet] Brasília: Ministério da Saúde; 2010 [acesso em 04 fev 2018]. Disponível: http://bvsms.saude.gov.br/bvs/publicacoes/diretrizes_do_nasf_nucleo.pdf.

6. Tesser CD. Núcleos de Apoio à Saúde da Família, seus potenciais e entraves: uma interpretação a partir da atenção primária à saúde. Interface (Botucatu). [Internet] 2016;21(62) [acesso em 22 mai 2017]. Disponível: http://dx.doi. org/10.1590/1807-57622015.0939.

7. Majid S, Foo S, Luyt B, Zhang X, Theng Y, Chang Y, et al. Adopting evidence-based practice in clinical decision making: nurse's perceptions, knowledge, and barriers. J. Med Libr Assoc. [Internet] 2011;99(3) [acesso em 15 mai 2017]. Disponível: https://dx.doi.org/10.3163\%2F1536-5050.99.3.010.

8. Barría-Pailaquilen RM. Practice Based on Evidence: An opportunity for quality care. Inv. Educ. Enferm. [Internet] 2013;31(2) [acesso em 15 dez 2017]. Disponível: https://aprendeenlinea.udea.edu.co/revistas/index.php/iee/article/ view/16009/14006.

9. Sackett DL. Trad. Ivan Carlquist. Medicina baseada em evidências: prática e ensino. 2 ed. Porto Alegre: Artmed; 2003.

10. Lacerda RA, Egry EY, Fonseca RMGS, Lopes NA, Nunes BK, Batista AO, et al. Práticas baseadas em evidências publicadas no Brasil: identificação e reflexão na área da prevenção em saúde humana. Rev. esc. enferm. USP. [Internet] 2012;46(5) [acesso em 15 dez 2017]. Disponível: http://dx.doi.org/10.1590/S0080-62342012000500028.

11. Lacerda RA, Nunes BK, Batista AO, Egry EY, Graziano KU, Angelo M, et al.

Práticas baseadas em evidências publicadas no Brasil: identificação e análise de suas vertentes e abordagens metodológicas. Rev. esc. enferm. USP. [Internet] 2011;45(3) [acesso em 25 mai 2017]. Disponível: http://dx.doi. org/10.1590/S0080-62342011000300033.

12. Pedrosa KKA, Oliveira ICM, Feijão AR, Machado RC. Enfermagem baseada em evidência: caracterização dos estudos no Brasil. Cogitare Enferm. [Internet] 2015;20(4) [acesso em 10 mai 2017]. Disponível: http://dx.doi.org/10.5380/ ce.v20i4.40768.

13. Chapecó (SC). Plano Municipal de Saúde de Chapecó 2014-2017. [Internet] Chapecó: Secretaria da Saúde; 2014. [acesso em 20 mai 2017]. Disponível: https://secsaude.chapeco.sc.gov.br/uploads/o-planejamento/2/plano-de-saudeii-ed-13052015.pdf.

14. Rospendowiski K, Alexandre NMC, Cornelio ME. Adaptação cultural para o Brasil e desempenho psicométrico do "Evidence-BasedPracticeQuestionnaire". Acta paul. enferm. [Internet] 2014;27(5) [acesso em 18 mai 2017]. Disponível: http://dx.doi.org/10.1590/1982-0194201400068. 
15. Silva TM, Costa LCM, Costa LOP. Evidence-Based Practice: a survey regarding behavior, knowledge, skills, resources, opinions and perceived barriers of Brazilian physical therapists from São Paulo state. Braz. J. Phys. Ther. [Internet] 2015;19(4) [acesso em 22 mai 2017]. Disponível: http://dx.doi.org/10.1590/bjpt-rbf.2014.0102.

16. Shafiei E, Baratimarnani A, Goharinezhad S, Kalhor R, Azmal M, et al. Nurses' perceptions of evidence-based practice: a quantitative study at a teaching hospital in Iran. Med. J. Islam. Repub. Iran. [Internet] 2014;28(1) [acesso em 22 mai 2017]. Disponível: https://www.ncbi.nlm.nih.gov/pubmed/25694993.

17. Weng Y, Kuo KN, Yang CY, Lo HL, Chen C, Chiu YW. Implementation of evidence-based practice across medical, nursing, pharmacological and allied healthcare professionals: a questionnaire survey in nationwide hospital settings. Implement Sci. [Internet] 2013;8(112) [acesso em 22 mai 2017]. Disponível: http://dx.doi.org/10.1186/1748-5908-8112.

18. Ministério da Saúde (BR). Autoavaliação para Melhoria do Acesso e da Qualidade de Atenção Básica - AMAQ. [Internet] Brasília: Ministério da Saúde; 2013 [acesso em 22 mai 2017]. Disponível: http://189.28.128.100/dab/docs/ portaldab/publicacoes/amaq_nasf.pdf.

19. Núcleo de Telessaúde da Universidade Federal Do Rio Grande Do Sul [Internet]. Porto Alegre: Sobre o Telessaúde RS: Quem somos? [acesso em 16 mai 2017]. Disponível: https://www.ufrgs.br/telessauders/sobre-o-telessauders/quemsomos/.

20. Cunha GT. A construção da clínica ampliada na atenção básica. 3 ed. São Paulo: Hucitec; 2010. p. 212.

21. da Silva ATC, de Aguiar ME, Winck K, Rodrigues KGW, Sato ME, Grisi SJFE, et al. Núcleos de Apoio à Saúde da Família: desafios e potencialidades na visão dos profissionais da Atenção Primária do Município de São Paulo, Brasil. Cad. Saúde Pública. [Internet] 2012;28(11) [acesso em 22 mai 2017]. Disponível: http://dx.doi.org/10.1590/S0102$311 \times 2012001100007$.

22. Dyniewicz AM. Análise das publicações dos enfermeiros assistenciais em periódicos nacionais. Rev. bras. enferm. [Internet] 2010;63(6) [acesso em 22 mai 2017]. Disponível: http://dx.doi.org/10.1590/S0034-71672010000600027.

23. Beltran P, Gonzalez-Torrente S, De Pedro-Gomez J, Morales-Asencio JM, Bennasar-Veny M, Perception of Spanish primary healthcare nurses about evidence-based clinical practice: a qualitative study. Int. Nurs. Rev. [Internet] 2014;61(1) [acesso em 22 mai 2017]. Disponível: http://dx.doi.org/10.1111/inr.12075.

24. Mallion J, Brooke J. Community- and hospital-based nurses implementation of evidence-based practice: are there any differences? Br. J. Community Nurs. [Internet] 2016;21(3) [acesso em 22 mai 2017]. Disponível: http://dx.doi. org/10.12968/bjcn.2016.21.3.148.

25. Patelarou AE, Patelarou E, Brokalaki H, Dafermos V, Thiel G, Melas CD, et al. Current evidence on the attitudes, knowledge and perceptions of nurses regarding evidence-based practice implementation in European community settings: a systematic review. J. Community Health Nurs. [Internet] 2013;30(4) [acesso em 22 mai 2017]. Disponível: http://dx.doi.org/10.1080/07370016.2013.838501. 